\title{
Die Entwicklung der Kontaktlinse in den letzten Jahrzehnten
}

\author{
Bruno Miller
}

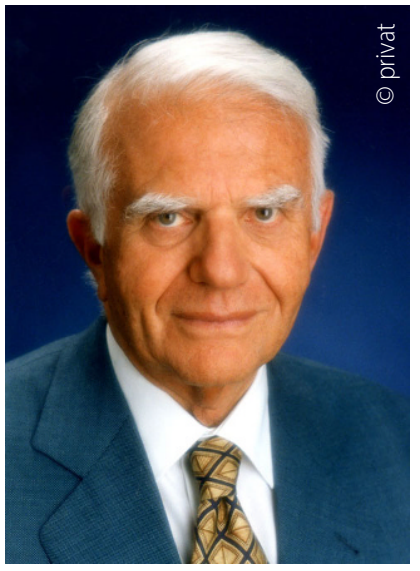

Die Idee der Kontaktlinse wurde durch Leonardo da Vinci und René Descartes vorbereitet. Die ersten als Korrektionsmittel verwendeten Haftschalen wurden 1888 von den Augenärzten Adolf E. Fick in Zürich und Eugen Kalt in Paris entwickelt. Bis 1920 beschränkte man sich auf die Anwendung beim Keratokonus und der hochgradigen Myopie.

1946 machte ich als junger Assistent an der Univ.Augenklinik in Innsbruck meine ersten Anpassversuche mit afokalen Corneosclerallinsen aus Glas, die die Firma Zeiss 1927 mit dem Ophthalmologen Heine herausgebracht hatte. Obrig in den USA und Györffy in Budapest verwendeten als erste Plexiglas (Anfang der 50er Jahre).

Wegweisend war dann die Einführung der Corneallinsen ohne haptischen Teil 1947 durch Tuohy. Diese Linsen wurden in einem Drehverfahren hergestellt und waren kleiner als der Hornhautdurchmesser. Diese schwimmende Kontaktlinse verbesserte den Trä-

Prof. MR. Dr. B. Miller $(\bowtie)$

Meraner Straße 3, 6020 Innsbruck, Österreich bruno@miller.at nenaustausch und hatte Vorteile für den Hornhautmetabolismus. Zur gleichen Zeit wurden solche Corneallinsen in Deutschland von Wöhlk und Söhnges entwickelt.

Entscheidend für die weitere Verbreitung von Kontaktlinsen war die Einführung der weichen Linse. 1955 entwickelten die Chemiker Lim und Wichterle in Prag ein Material, das ursprünglich als Glaskörperersatz geplant war. Das Hemamaterial wurde im trockenen $\mathrm{Zu}$ stand als Button im Drehverfahren bearbeitet. 1965 kaufte die Firma Bausch \& Lomb das tschechische Patent und verfeinerte die Herstellung durch das Schleudergussverfahren.

Für die bessere Verträglichkeit, das geringere Fremdkörpergefühl und die geringere Belastung des Hornhautstoffwechsels waren die Fortentwicklung der Materialien und der Linsengeometrie erforderlich. Entscheidend dafür war die Sauerstoffdurchlässigkeit, die durch die Diffusionskonstante (DK) bestimmt ist. Harte Kontaktlinsen aus PMMA sind formstabil und pflegeleicht, jedoch sauerstoffundurchlässig, CAB hat einen DK von 4-8, Silikonlinsen haben zwar eine höhere Sauerstoffpermeabilität, jedoch eine schlechte Benetzbarkeit. Die weichen Materialien erreichen eine höhere Sauerstoffpermeabilität.

Bausch \& Lomb und Ciba-Vision entwickelten bis 1999 Weichlinsenmaterialien, die sich auch für verlängertes Tragen eigneten. Diese haben trotz des niedrigeren Wassergehaltes und der hohen Sauerstofftransmission eine gleichbleibende gute Benetzung.

Bei den formstabilen Linsen machte die Entwicklung Fortschritte auf dem Gebiet der Linsengeometrie: die ersten harten Linsen waren einkurvig, später zwei- und dreikurvig mit verblendeten Übergangszonen. Verbesserte Messmethoden der Hornhautradien, und später aus diesen Messungen erstellte Computerprogramme, ermöglichten die Herstellung asphärischer harter Linsen mit unterschiedlichen Ex- 
zentrizitäten und damit die exakte Anpassung an die Hornhautoberfläche. Eine weitere Verbesserung der Hornhautvermessung brachte die Videokeratoskopie 1985. Dadurch wurden auch dreidimensionale Darstellungen möglich. Fortentwickelte Computerprogramme geben bei der Anpassung von harten Speziallinsen relativ exakte Vorschläge für die Linsengeometrie. Weitere Kriterien sind jedoch für die Linsenanpassung sehr wichtig: Tränenfilm, pH-Wert der Tränen, Lidspaltenweite, Oberlidspannung und Linsendurchmesser.

Grundsätzlich liegt die Gefahr in der Störung der Hornhautmorphologie und des Hornhautstoffwechsels durch die Barriere der Kontaktlinse. Diese ist bei den weichen Linsen höher anzusetzen, da die Weichlinsen einen größeren Durchmesser als die Hornhaut haben. Die harten Linsen hingegen sind kleiner und lassen den Hornhautrand frei. In der Folge kann es bei übertriebenem Linsentragen zu Mikrozysten, verringerter Sensibilität, limbalen Gefäßneubildungen, Hornhautstippung, Descemetfaltenbildung oder sogar tiefer Vascularisation und Narbenbildung kommen. Neben der exakten Anpassung ist auch der Tränenfilm von essentieller Bedeutung, sowohl in der Menge als auch in der Zusammensetzung. Ablagerungen an der Linsenoberfläche können durch fortentwickelte Verfahren besser diagnostiziert werden (Phasenkontrast, Interferenzkontrast). Das Rasterelektronenmikroskop gestattet eine quantitative Analyse der Elemente an der Oberfläche. Durch elektrophoretische Untersuchungen lassen sich die Hauptfraktionen der Tränenproteine feststellen, das anodische Albumin, das kathodische Lysozym.

Die Entwicklung der Kontaktlinsenpflege begründete einen ganzen Industriezweig. Diese hygienischen Maßnahmen sollen den Augenschäden durch Bakterien, Viren und Pilzen entgegenwirken. Anorganische Ablagerungen werden durch Kompexbildner entfernt, organische Ablagerungen werden biochemisch mittels Enzymen abgebaut, der chemische Abbau erfolgt durch Oxydasen, die Desorption durch Tenside und die Verhinderung von bakteriellem Befall durch thermische Desinfektion oder Sterilisierung. Die Hauptquelle der bakteriellen Kontamination ist die tägliche Manipulation und die Verunreinigung der Behälter. Die Einführung der sog. Wegwerflinsen oder Tageslinsen wirkt dem entgegen.
Auf dem Sektor der formstabilen Spaziallinsen sind die verschiedenen torischen Linsen $\mathrm{zu}$ erwähnen, die es ermöglichen, praktisch jeden Astigmatismus zu korrigieren, sowie die Keratokonus-Linsen. Die Versorgung der Patienten mit Keratokonus ist eine schwierige aber auch dankbare Aufgabe: Der irreguläre Astigmatismus kann mit einer Brille nicht korrigiert werden. Mit der speziellen Anpassung harter Keratokonus-Linsen kann man jedoch ein gutes Sehvermögen wiederherstellen. Nach dem operativen Eingriff einer Keratoplastik oder refraktiven Chirurgie kann es erforderlich sein, Linsen mit negativer Exzentrizität anzupassen.

Da viele Kontaktlinsenträger auch ins Presbyopenalter kommen, gewinnen die Bifokal- oder Multifokallinsen immer mehr an Bedeutung. Heute kommen zumeist zwei Prinzipen zum Tragen: Bifolinsen für simultanes Sehen oder das alternierende Prinzip mit Prismenballast.

In den letzten Jahren hat die refraktive Chirurgie stark an Bedeutung gewonnen und damit einen Teil der Kontaktlinsenträger verdrängt. Der in Contactologie erfahrene Ophthalmologe wird jedoch nach wie vor ein weites Betätigungsfeld haben, insbesondere in der Anpassung von Speziallinsen bei hochgradiger Fehlsichtigkeit, Astigmatismus, Keratokonus, nach Keratoplastik oder als Therapielinsen. Die Anpassung und Kontrolle dieser Linsen gehört nach wie vor in augenärztliche Hände.

Nach dem erfolgreichen Abschluss meines Medizinstudiums zu Kriegsende in Innsbruck war ich fünf Jahre lang an der Universitäts-Augenklinik in Innsbruck tätig. Anschließend habe ich 25 Jahre lang eines der größten Optikergeschäfte, die Firma OptikMiller, geführt. 1961 habe ich eine eigene Praxis eröffnet, wo ich als Ophthalmologe und Kontaktologe viele Jahre tätig war. Ich habe über 35 eigene Arbeiten in der Contactologia, in den Klinischen Monatsblättern für Augenheilkunde und bei der International Society of Contact Lens Specialists veröffentlich. Über viele Jahre war ich in der ECLSO (European Contact Lens Society of Ophthalmologists) tätig.

Interessenkonflikt B. Miller gibt an, dass kein Interessenkonflikt besteht. 